\title{
An FPGA based Minutiae Extraction System for Fingerprint Recognition
}

\author{
Yousra Wakil \\ NED University of \\ Engg and Technology \\ University Road, \\ Karachi, Pakistan
}

\author{
Sehar Gul Tariq \\ NED University of \\ Engg and Technology \\ University Road, \\ Karachi, Pakistan
}

\author{
Aniza Humayun \\ NED University of \\ Engg and Technology \\ University Road, \\ Karachi, Pakistan
}

\author{
Naeem Abbas \\ National University of \\ Sciences and \\ Technology \\ Karsaz Road, \\ Karachi, Pakistan
}

\begin{abstract}
Fingerprint holds a higher priority among other identification techniques such as passwords, keys and ID cards. Biometric systems which include fingerprint recognition, is essential as every individual has a unique fingerprint pattern. An efficient approach towards fingerprint recognition is through the implementation of image processing techniques that can be performed efficiently along with embedded systems such as FPGA for fast execution. This paper presents FPGA based architecture for fingerprint recognition by using Xilinx System Generator that efficiently implements the highly demanding image processing techniques along with crossing number method of ridge feature based technique. Moreover minutiae matching algorithm and input from sensor for real time authentication of fingerprints can further be implemented on this system.
\end{abstract}

\section{Keywords}

Fingerprint images, minutiae extraction, Xilinx System Generator.

\section{INTRODUCTION}

Fingerprint distinguishes from person to person therefore they can be regarded as a source of identifying a person, as no two person have the same fingerprints therefore recognition through fingerprints is becoming a rapidly evolving technology which is widely being used in recognition criminals, identifying corpse, national ID cards, defense, passport control, social security, medical, data security and in a number of fields. Since this the process must be fast and efficient to perform the job, many algorithms have been developed. However a majority of these algorithms are unable to identify a fingerprint image on real time basis due to high sensitivity of errors which are caused by the roughness of skin, damaged part due to some injury, misalignment of finger on the sensors and many more factors that causes problems, to overcome these problem one advanced technique is employed in recent years which is being discuss in this paper.

The minutiae based algorithm provides efficient fingerprint recognition. It extracts features called minutiae points which includes ridges and bifurcation from the fingerprint image and compare these points with the already stored fingerprint image. The extraction and comparison of minutiae features requires efficient algorithms for efficient processing of the fingerprint image, therefore we used cross number method, at the same time the process must be fast that can handle number of users so FPGA is used as hardware platform, as it provides parallel processing and enables real time processing. However FPGA based circuits requires high design time efforts. To reduce the design time, we used High-level synthesis tool, Xilinx system generator. Xilinx system generator is integrated with MATLAB
Simulink and it generates a Verilog code in correspondence to design or module created on MATLAB Simulink to configure FPGA[1].

The paper is divided into following sections: section II explains the design and the working of the system. Section III discusses the results. Section IV concludes the paper followed by the key references used in this work.

\section{SYSTEM MODEL}

The model is designed using Xilinx System Generator (Simulink Block) MATLAB.

The work is divided into 5 parts. In first part a grayscale image from memory is acquired by image acquisition using Simulink MATLAB. The second part is to remove noise from the image by using image enhancement technique. The third part is to convert the grayscale image into a binary image by using image binarization technique. The

Fourth step is to make the binary image as thin as possible so that each pixel represents a bit value using image thinning. Finally, the last part is to extract the features of a thinned fingerprint image i.e. the ridge endings and ridge bifurcation points. The overall block diagram of the proposed system is given below.

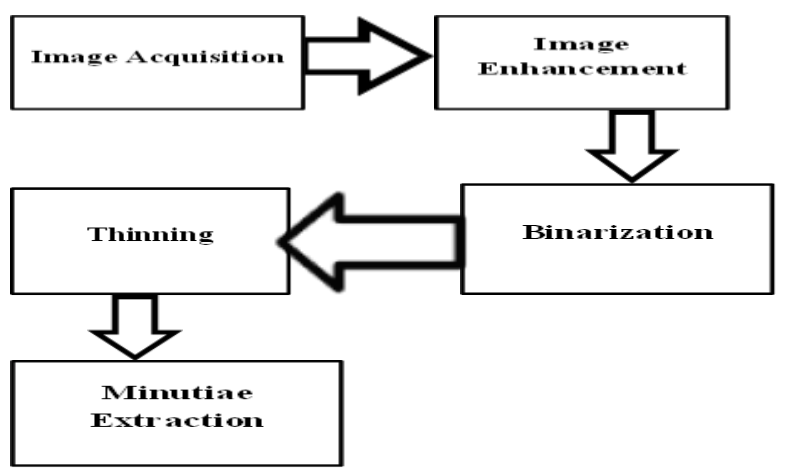

Fig 1: Block Diagram of the Proposed Model

\subsection{Image Acquisition}

In this process the gray scale image is acquired from the memory and stored for further processing. Image acquisition consists of following pre-processing and post-processing blocks:

\section{Image Pre-processing:}

Image pre-processing consists of the following blocks:

- $\quad$ Resize: The resize block is used to enlarge or shrink image size. It also sets input dimensions for an image and interpolation. 
- Convert 2-D to 1-D: The convert 2-D to 1-D block is used to reshape an M-by-N matrix input to a 1-D vector with length $\mathrm{M}^{*} \mathrm{~N}$.

- Frame conversion and un-buffer: It converts the data that is represented in column into rows. Fig 2.1 (a) shows image pre-processing.

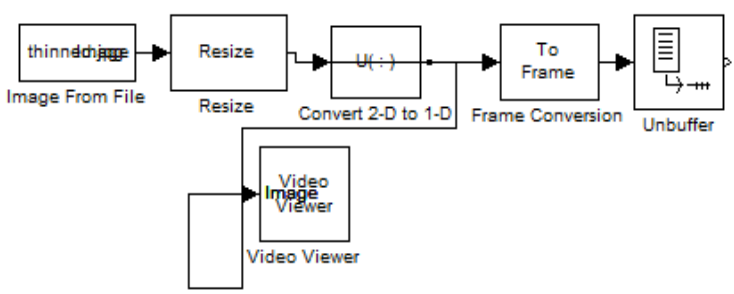

Fig 2.1(a) In Image pre-processing, the image is being acquired and after converting from 2D to 1D it is displayed[5].

\section{Image Post-Processing:}

Image post- processing helps to recreate image from 1-D array. It consists of the following blocks:

- Data Type Conversion: It converts image signal to unsigned integer format.

- Buffer: The Buffer block always performs frame-based processing. The block redistributes the data in each column of the input to produce an output with a different frame size. Buffering a signal to a larger frame size yields an output with a slower frame rate than the input.

- Convert 1-D to 2-D: It converts 1-D image signal to 2-D image matrix.

- Sink: It is used to display the output image back on the monitor. The Fig 2.1(b) shows image post-processing.

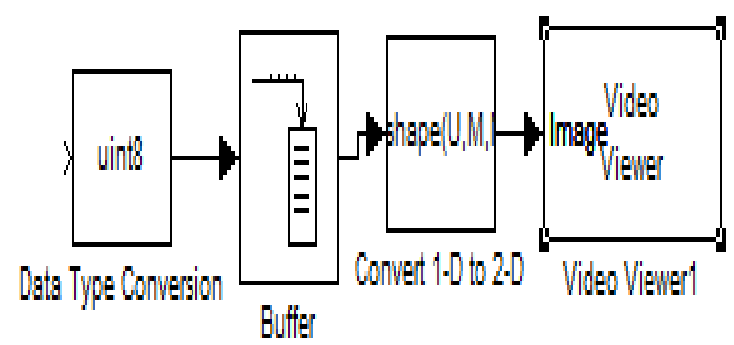

Fig 2.1(b) In Image Post-processing, image type is converted to unsigned integer, Buffering reduces the frame rate of output image after that image is again converted into 2D.[5]

The next section explains the removal of noise from the image.

\subsection{Image Enhancement}

Image enhancement is a technique used for the elimination of noise and distortion, making the image sharper for better visualization as well as improving the edges so that further processing become easy.

The gray scale fingerprint image is enhanced by adding some illumination to it. For this purpose an AddSub block is used that adds a constant fixed point value 5 to the image as shown in fig 2.2 .
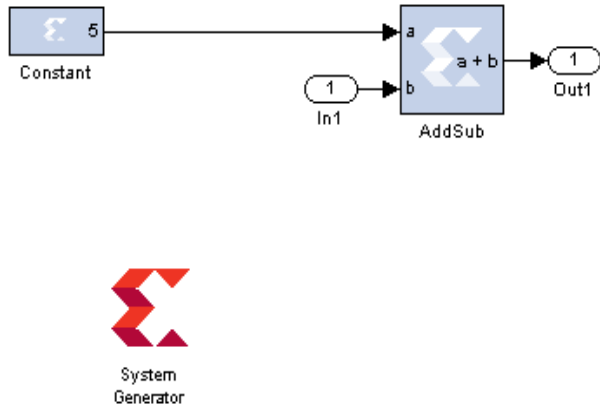

Fig 2.2 Image Enhancement is used to make image enhance and reduce distortion and noise for sharper results[5].

\subsection{Binarization}

The gray scale fingerprint image is then converted into a binary image. The image intensity in binary image consists of the following two values:

a) Black representing the ridges

b) White representing the valleys and background

Binarization of an image is shown in Fig 2.3. Here the technique that is used to obtain a binary image is thresholding. A relational block is used which is used to implement a comparator. It is used to compare the pixels of the gray scale image with the threshold value which is set as ' 120 '. The output of the relational block is then fed to the MUX block which implements a multiplexer. If the pixels of the image are above the threshold value so the pixels are set as ' 0 ' otherwise they are set to ' 1 '
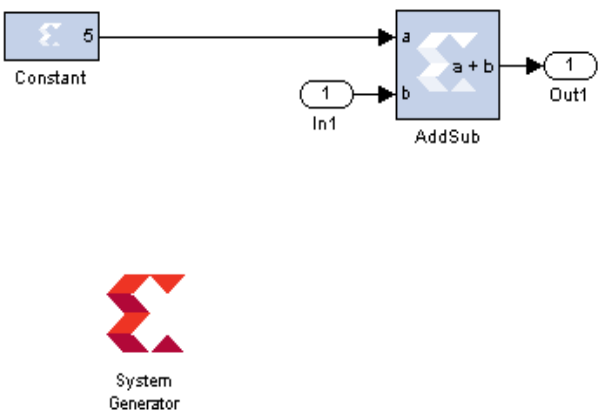

Fig 2.3 Image Binarization is used to convert a gray scale image into binary image by setting a specific threshold

\subsection{Thinning}

After binarization image thinning algorithm is applied. Thinning algorithm is used to remove selected foreground pixels from binary images. It is used in many applications as it makes the object representation as thin as possible so that object width is represented by a single pixel.

The techniques used for image thinning are Perwitt edge detection and Sobel operator [6] but this paper contains Perwitt edge detection technique. The edge in a fingerprint is characterized by its length, slope angle and coordinate of slope midpoint. Edges are of two types a ramp edge, in which the intense values change slowly, and a step edge, in which the intense values change abruptly. The convolution mask of Perwitt edge detection is shown in Fig 2.4(a). 


\begin{tabular}{|l|l|l|}
\hline-1 & 0 & +1 \\
\hline-1 & 0 & +1 \\
\hline-1 & 0 & +1 \\
\hline \multicolumn{4}{|c}{$\mathrm{Gx}$}
\end{tabular}

\begin{tabular}{|l|l|l|}
\hline+1 & +1 & +1 \\
\hline 0 & 0 & 0 \\
\hline-1 & -1 & -1 \\
\hline \multicolumn{4}{|c}{ Gy }
\end{tabular}

Fig 2.4 (a) Convolution mask of edge detection (Prewitt)[3]

In order to get a convolute image the $\mathrm{x}$ and $\mathrm{y}$ coordinate are summed up together so that the value can be used in the below formula. This can be shown mathematically as,

$$
|G|=\left|G_{x}\right|+\left|G_{y}\right|
$$

Mathematically gradient can be computed by the equation given below:

$$
|G|=\left(G_{x}^{2}+G_{y}^{2}\right)^{\frac{1}{2}}
$$

- Horizontal filter gradient for Perwitt Operator:

The Horizontal gradient of an image is computed by moving horizontal kernel for Perwitt Edge Detection over an image, as shown in Fig 2.4(b).

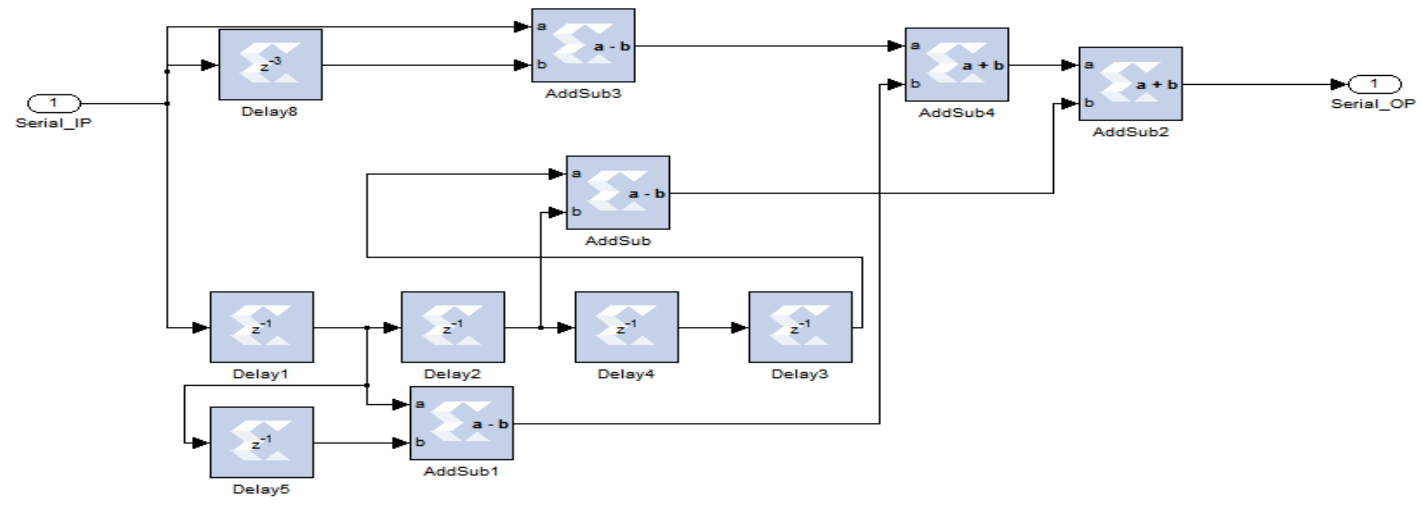

Fig 2.4 (b) Horizontal gradient filter (Perwitt) with number of delays and AddSub blocks [4]

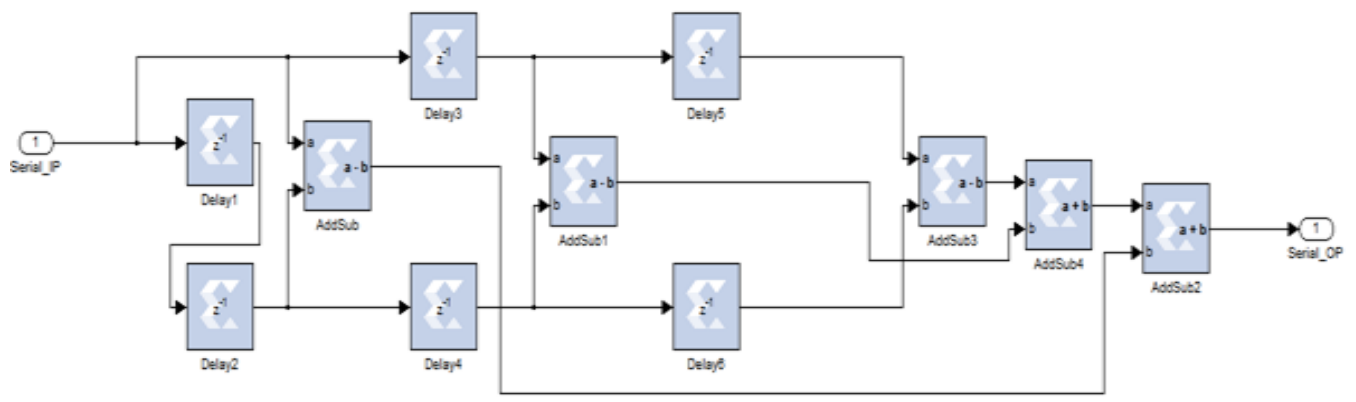

Fig 2.4 (c) Vertical gradient filter (Perwitt) with number of delays and AddSub blocks [4]

\section{- Vertical filter gradient for Perwitt Operator:}

The Vertical gradient of an image is computed by moving vertical mask for Perwitt Edge Detection over an image shown in fig 2.4(c)

\section{By adding together horizontal and vertical gradients}

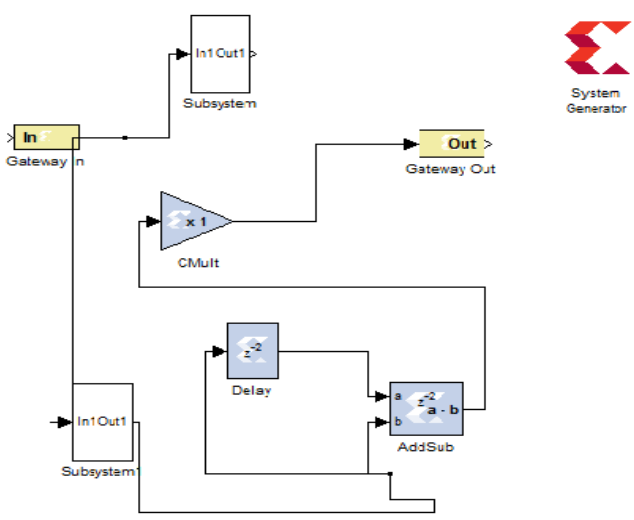

Fig 2.4 (d) Image Thinning by combining horizontal and vertical Perwitt operators

\subsection{Minutiae Extraction}

After the process of fingerprint image thinning, minutiae extraction algorithm is implemented. In minutiae extraction algorithm features of a thinned fingerprint image are extracted i.e. the ridge endings and ridge bifurcations points. Ridge ending (it is also identified as a valley skeleton bifurcation point);

- Ridge bifurcation (it is also identified as valley skeleton end point);

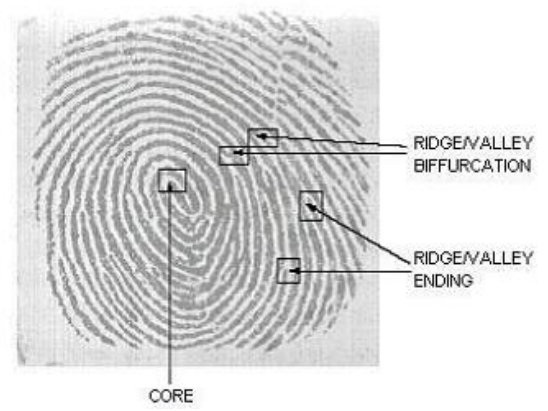

Fig 2.5 (a) Fingerprint Image Structure 


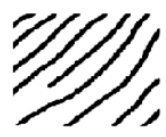

Ridge ending

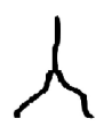

Bifurcation

\section{Fig 2.5 (b) Ridge ending and Bifurcation}

In minutiae extraction the thinned image is taken as input which is then being masked with the $3 \times 3$ window, which contain ones and zeroes according to the conditions of ridge ending and bifurcations, this window is passed over the thinned image whose each pixel contain one bit value which is then being $\mathrm{XNOR}$ with the $3 \times 3$ window[9] so that ' 1 ' is obtained if it exactly matches, in this way a number of windows is passed over the image with different values to extract the different features of the fingerprint.[8]

After that a counter is added that checks the image is being masked completely if so it passes ' 1 ' at the input of an AND gate which becomes high when it receives a high input from the counter as well as from the matched pixel, finally the values are save in a single port Ram, which are actually the pixel number.

These pixel numbers are then divided by the number of pixel in a row/column, the obtained quotient is the $\mathrm{x}$-coordinate while the remainder is the y-coordinate, which is then plotted on the processed image. In these way minutiae points are extracted. The design for minutiae extraction algorithm is shown in fig 2.5 (c).

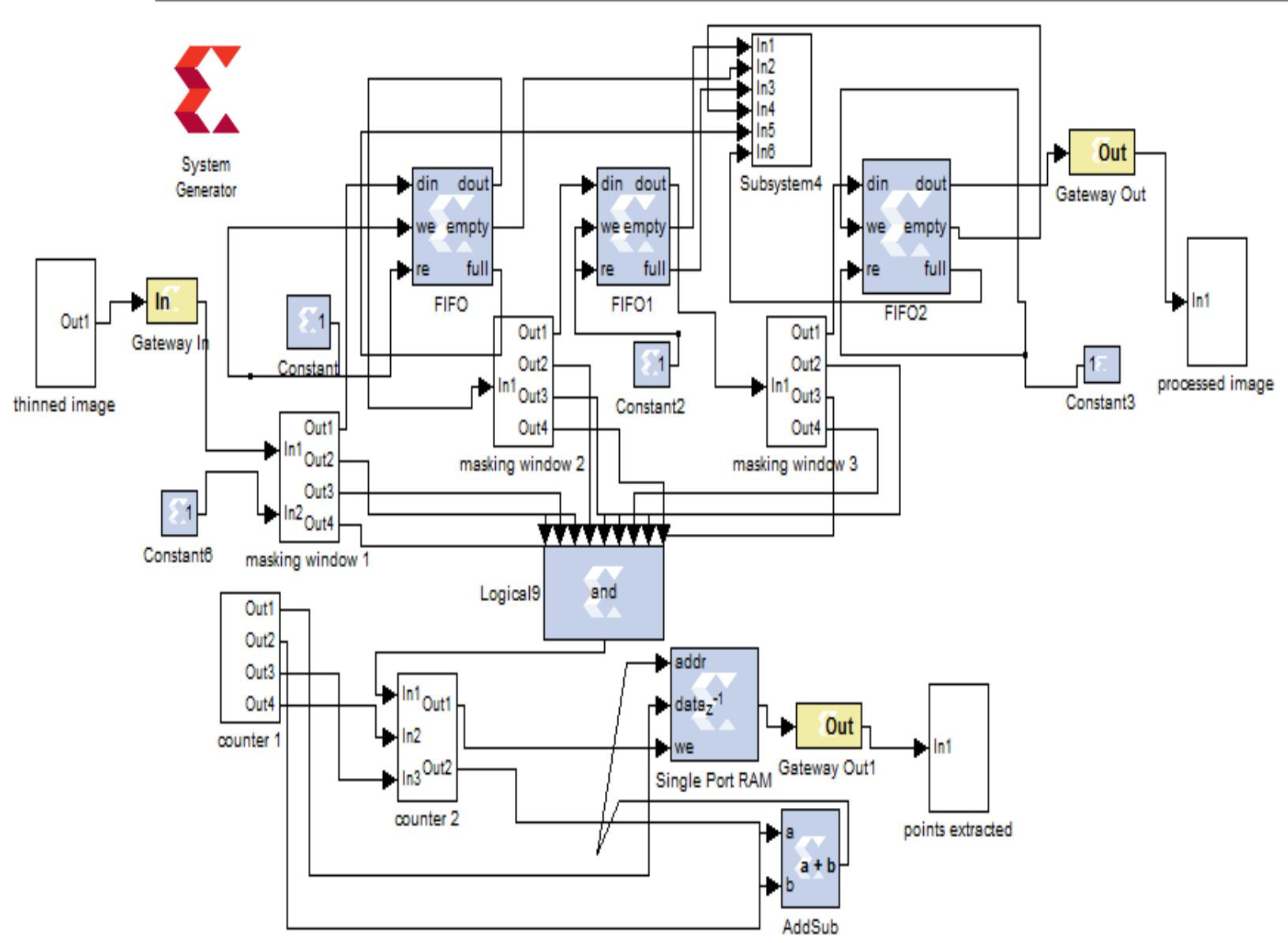

Fig 2.5 (c) Minutiae Extraction by masking the image with 3x3 windows

\section{RESULTS}

The experimental results for FPGA based fingerprint matching algorithm are shown below: Fig 3(a) shows grayscale fingerprint image as input.

Fig 3(b) shows the result for image enhancement.

Fig 3(c) shows the result for image binarization.

Fig 3(d) shows the result for image thinning.

Fig 3(e) shows the image after minutiae extraction.

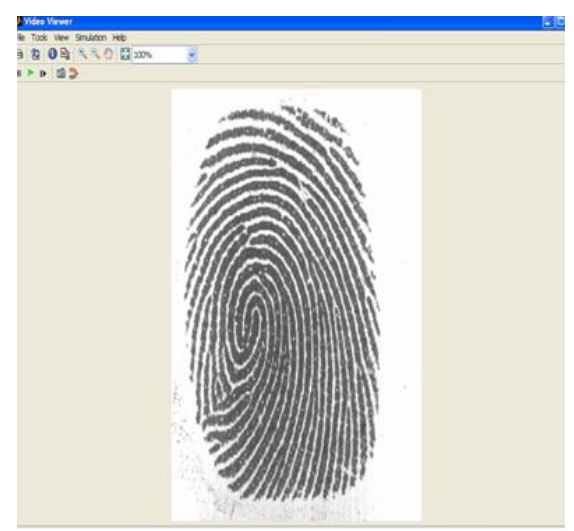

Fig 3(a) Input Image 


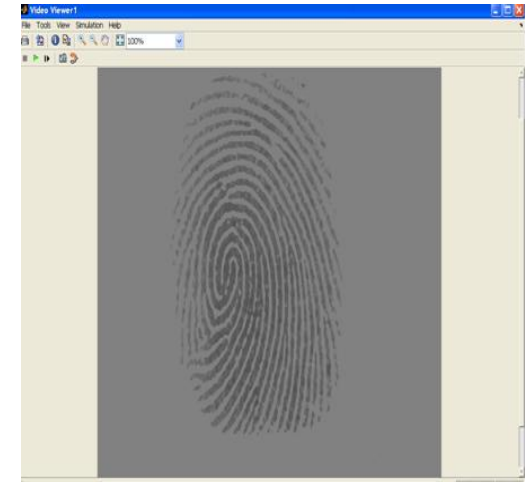

Fig 3(b) Enhanced Image

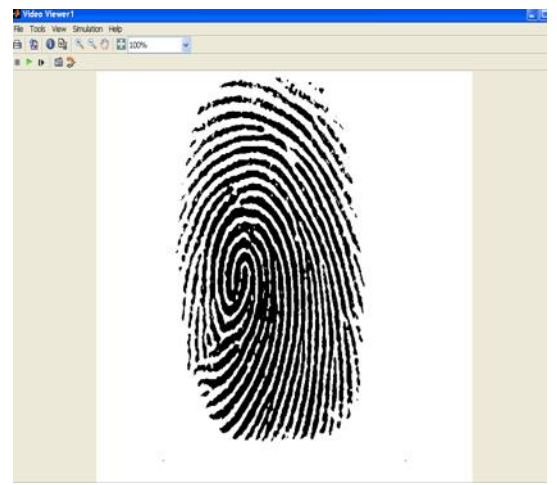

Fig 3(c) Binarized Image

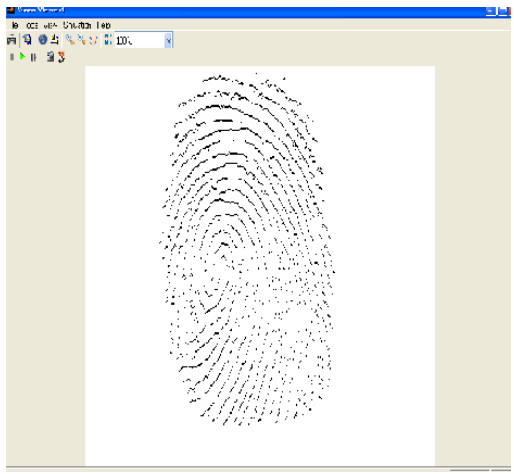

Fig 3(d) Thinned Image

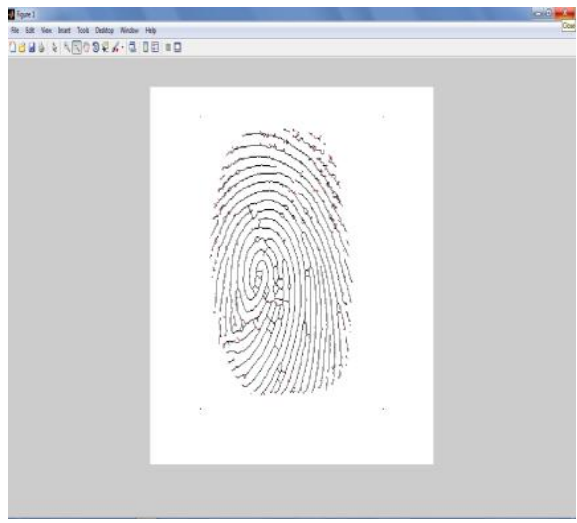

Fig 3(e) Image after Minutiae Extraction

\section{CONCLUSION AND FUTURE WORK}

The image processing algorithm is successfully implemented till fingerprint minutiae extraction using Xilinx System Generator. Xilinx System Generator offers a model based design for processing. It is considered to be an advantageous option for development in more easy and comfortable way than permitted by VHDL or Verilog Hardware Description Language. Another advantage of Xilinx System Generator is that is supports software simulation, and also generates necessary files with the parallelism, speed, robust and automatic area minimization for implementation on all Xilinx FPGA's.

Since the algorithm is so far implemented till fingerprint minutiae extraction further minutiae matching algorithm has to be implemented using Xilinx System Generator which can be further implemented on all Xilinx FPGA kits for fast and efficient real time processing. This FPGA based fingerprint matching gives high accuracy and can be used for high security systems.

\section{REFERENCES}

[1] Mrs.S. Allin christe, Mr.M.vignesh, Dr.A.Kandaswamy "An efficient fpga implementation of mri image filtering and tumour characterization using xilinx system generator", International Journal of VLSI design \& Communication Systems (VLSICS) Vol.2, No.4, December 2011.

[2] Neha P.Raut, Prof A.V.Gokhale "FPGA implementation of image processing algorithms using Xilinx System Generator", IOSR Journal of VLSI and Signal Processing (IOSR-JVSP) Volume 2, Issue 4 (May- June 2013), PP 2636, e-ISSN: 2319-4200, p-ISSN No.:2319-4197.

[3] T. Mahalakshmi and R.Muthaiah "Vlsi Implementation of Edge Detection for Images", Research Journal of Applied Sciences, Engineering and Technology ISSN:@ Maxwell Scientific Organization, 2012.

[4] Swapnil G. Kavitkar, Prashant L. Paikrao "FPGA based Image Feature Extraction Using Xilinx System Generator", Swapnil G. Kavitkar et al, / (IJCSIT) International Journal of Computer Science and Information Technologies, Vol. 5 (3) , 2014, 3743-3747.

[5] Alareqi Mohammed, Elgouri Rachid and Hlou Laamari "High Level FPGA Modeling for Image Processing Algorithms Using Xilinx System Generator", International Journal of Computer Science and Telecommunications [Volume 5, Issue 6, June 2014].

[6] Ravi.s1 Abdul Rahim.B, Fahimuddin shaik "FPGA Based Design and Implementation of Image Edge Detection Using Xilinx System Generator", International Journal of Engineering Trends and Technology (IJETT) - Volume 4 Issue 10 - Oct 2013.

[7] Manvjeet Kaur, Mukhwinder Singh, Akshay Girdhar, and Parvinder S. Sandhu Fingerprint Verification System using Minutiae Extraction Technique World Academy of Science, Engineering and Technology

[8] Sunny Arief Sudiro and Rudi Trisno Yuwono adaptable fingerprint minutiae extraction algorithm based-on crossing number method for hardware implementation using fpga device, International Journal of Computer Science, Engineering and Information Technology (IJCSEIT), Vol.2, No.3, June 2012

[9] Roli Bansal, Priti Sehgal and Punam Bedi Minutiae Extraction from Fingerprint Images, IJCSI International Journal of Computer Science Issues, Vol. 8, Issue 5, No 3, September 2011 